\title{
RELATIVISTIC AHARONOV-BOHM-COULOMB PROBLEM
}

\author{
C. R. Hagen and D. K. Park* \\ Department of Physics and Astronomy \\ University of Rochester \\ Rochester, NY 14627, USA
}

\begin{abstract}
The Aharonov-Bohm effect is analyzed for a spin- $1 / 2$ particle in the case that a $1 / r$ potential is present. Scalar and vector couplings are each considered. It is found that the approach in which the flux tube is given a finite radius that is taken to zero only after a matching of boundary conditions does not give physically meaningful results. Specifically, the operations of taking the limit of zero flux tube radius and the Galilean limit do not commute. Thus there appears to be no satisfactory solution of the relativistic AharonovBohm-Coulomb problem using the finite radius flux tube method.
\end{abstract}

* On leave from Dept. of Physics, Kyung Nam University, Masan, 631-701, Korea 


\section{Introduction}

Since the time of its discovery the Aharonov-Bohm (AB) effect [1] has been the object of considerable attention at both theoretical and experimental levels [2]. Much of the recent attention given to this phenomenon has been associated with the fact that for spinless nonrelativistic particles it allows an interpretation in which the interaction can be eliminated provided that fractional statistics are introduced [3]. In fact it has been suggested that high- $T_{c}$ superconductivity phenomena may be best understood using fractional statistics. That interpretation, however, cannot be maintained when one considers spin effects in the $\mathrm{AB}$ problem, since the latter has the property of introducing a delta-function potential into the Hamiltonian (i.e., the Zeeman interaction of the spin with the magnetic field). This term breaks an essential symmetry in the fractional statistics approach, namely the

invariance of the theory under translation of the flux parameter by an integer. The spin- $\frac{1}{2}$ AB problem has been discussed extensively by different methods. Gerbert [4] examined the problem from a mathematical point of view by the self-adjoint extension approach and concluded that an arbitrary combination of the regular and singular solutions could contribute to the wave function provided that

$$
|m+\alpha|<1
$$

where $\alpha$ is the flux parameter. The same problem was subsequently considered by one of us [5] in the framework of a more physical model. Specifically, the magnetic field in the Zeeman interaction term was defined in that approach to be the zero radius limit of a flux tube of finite radius, i.e., is proportional to

$$
\lim _{R \rightarrow 0} \frac{\alpha}{R} \delta(r-R)
$$

From the boundary conditions at $r=R$ it was then found that only the singular solution of the Schrödinger equation could contribute to the radial wave equation for the case

$$
\begin{aligned}
& |m|+|m+\alpha|=-\alpha s \\
& |m+\alpha|<1
\end{aligned}
$$

where $s$ is twice the spin projection. 
In order to achieve a physical realization of the self-adjoint extension method some authors [6] have recently considered the possibility of including strongly repulsive potentials inside the flux tube. However, it has been noted [7] that such calculations within the framework of the Dirac equation tend not to be reliable because of the occurrence of Klein's paradox. To avoid this difficulty the solution of the spin- $\frac{1}{2}$ Aharonov-Bohm-Coulomb $(\mathrm{ABC})$ problem [7] was obtained within the framework of the Galilean theory [8] which is free of such complications. It was then found that with the inclusion of the Coulomb potential, the range of flux parameter for which singular solutions are allowed is only half as large as that in the pure $\mathrm{AB}$ case, namely,

$$
|m+\alpha|<\frac{1}{2}
$$

Subsequently it was shown [9] that the self-adjoint extension method also gives the condition (1.4) for the occurrence of singular solutions.

The goal of this paper is to carry out an analysis of the $\mathrm{ABC}$ problem without recourse to the Galilean limit. It should be noted at the outset, however, that there are at least two ways in which a Coulomb potential can be included in the relativistic AB problem such that the same Galilean limit is obtained. Thus the analysis presented here considers both the scalar coupling defined by

$$
M \rightarrow M+\xi / r
$$

(where $M$ is the mass of the spin- $\frac{1}{2}$ particle and $\xi / r$ is the Coulomb potential) as well as the vector coupling

$$
E \rightarrow E-\xi / r
$$

Furthermore, two different realizations of the Coulomb potential within the flux tube are considered in this paper, one continuous $\left(V_{c}\right)$ and one discontinuous $\left(V_{d}\right)$. They are parametrized as

$$
\begin{aligned}
V_{c}(r) & =\frac{\xi}{r} \theta(r-R)+\frac{\xi}{R} \theta(R-r) \\
V_{d}(r) & =\frac{\xi}{r} \theta(r-R)
\end{aligned}
$$


where $\theta(x)$ is the usual step function

$$
\theta(x)=\frac{1}{2}\left(1+\frac{x}{|x|}\right) .
$$

In secs. 2 and 3 the Dirac equation of the ABC problem with a scalar coupling is solved explicitly, first for the case of the continuous potential (1.7a) and subsequently for the discontinuous one (1.7b). The comparisons between the relativistic AB and Galilean ABC problems are discussed in detail. Corresponding results for the vector coupling of the Coulomb potential are presented in sec. 4. A concluding section compares results in the Galilean and relativistic ABC problems and makes some general observations concerning the significance of the results obtained.

\section{Scalar Coupling of the Continuous Coulomb Potential}

In this section the relativistic $\mathrm{ABC}$ problem is analyzed for the case in which the Coulomb potential (1.7a) is included in the Dirac equation by means of a scalar interaction. Thus the relevant equation is

$$
\beta\{[M+V(r)]+\vec{\gamma} \cdot \vec{\Pi}\} \psi=E \psi
$$

where $\Pi_{i}=-i \partial_{i}-e A_{i}$ with $A_{i}$ the usual AB potential

$$
e A_{i}= \begin{cases}\frac{\alpha \epsilon_{i j} r_{j}}{r^{2}} & r>R \\ 0 & r<R\end{cases}
$$

A convenient choice for the matrices in $(2.1)$ is [5]

$$
\begin{aligned}
& \beta=\sigma_{3} \\
& \beta \gamma_{i}=\left(\sigma_{1}, s \sigma_{2}\right)
\end{aligned}
$$

where the $\sigma$ 's are the usual Pauli matrices and $s= \pm 1$ is the spin projection parameter of Eq. (1.3).

The second order equation implied by (2.1) is obtained by applying the matrix operator $[(M+V(r))+\beta E-\vec{\gamma} \cdot \vec{\Pi}] \beta$. The result is

$$
\begin{aligned}
\left\{\frac{\partial^{2}}{\partial r^{2}}+\frac{1}{r} \frac{\partial}{\partial r}\right. & +\frac{1}{r^{2}}\left(\frac{\partial}{\partial \phi}+i \alpha\right)^{2} \\
& \left.+E^{2}-[M+V(r)]^{2}+e s H \sigma_{3}+E_{1} \sigma_{2}-s E_{2} \sigma_{1}\right\} \psi=0
\end{aligned}
$$


where $H$ is the magnetic field

$$
e s H=-\frac{\alpha s}{R} \delta(r-R)
$$

and $E_{i}$ is the electric field

$$
E_{i}=-\partial_{i} V(r)
$$

Using (1.7a) one finds that

$$
E_{1} \pm i s E_{2}=\frac{\xi}{r^{2}} e^{ \pm i s \phi} \theta(r-R)
$$

so that (2.4) becomes

$$
\begin{gathered}
\left\{\frac{\partial^{2}}{\partial r^{2}}+\frac{1}{r} \frac{\partial}{\partial r}+\frac{1}{r^{2}}\left(\frac{\partial}{\partial \phi}+i \alpha\right)^{2}+E^{2}-\left[M+\frac{\xi}{r} \theta(r-R)+\frac{\xi}{R} \theta(R-r)\right]^{2}\right. \\
\left.+e s H \sigma_{3}\right\} \psi=-\frac{\xi}{r^{2}}\left(\begin{array}{cc}
0 & -i e^{-i s \phi} \\
i e^{i s \phi} & 0
\end{array}\right) \theta(r-R) \psi
\end{gathered}
$$

If one defines

$$
\psi=\left(\begin{array}{c}
\psi_{1} \\
\psi_{2}
\end{array}\right)=\left(\begin{array}{c}
\sum_{m} f_{m}(r) e^{i m \phi} \\
\sum_{m} g_{m}(r) e^{i(m+s) \phi}
\end{array}\right)
$$

Eq. (2.8) becomes

$$
\begin{aligned}
\left\{\frac{d^{2}}{d r^{2}}\right. & +\frac{1}{r} \frac{d}{d r}-\frac{(m+\alpha)^{2}}{r^{2}}+E^{2}-\left[M+\frac{\xi}{r} \theta(r-R)+\frac{\xi}{R} \theta(R-r)\right]^{2} \\
& \left.-\frac{\frac{1}{2}+s(m+\alpha)}{r^{2}}\left(1-\sigma_{3}\right)+\frac{\xi}{r^{2}} \sigma_{2} \theta(r-R)+e s H \sigma_{3}\right\}\left(\begin{array}{l}
f_{m} \\
g_{m}
\end{array}\right)=0
\end{aligned}
$$

From (2.10) the equation governing the inside region $(r<R)$ is seen to be

$$
\left[\frac{d^{2}}{d r^{2}}+\frac{1}{r} \frac{d}{d r}-\frac{m^{2}}{r^{2}}+E^{2}-\left(M+\frac{\xi}{R}\right)^{2}\right] f_{m}=0
$$

whose regular solution is

$$
f_{m}^{i n}(r)=J_{|m|}\left(k_{0} r\right)
$$

where

$$
k_{0}^{2}=E^{2}-\left(M+\frac{\xi}{R}\right)^{2}
$$


In the outside region $(r>R)$ Eq. (2.10) becomes

$$
\left[\frac{d^{2}}{d r^{2}}+\frac{1}{r} \frac{d}{d r}-\frac{\eta+(m+\alpha)^{2}}{r^{2}}+E^{2}-\left(M+\frac{\xi}{r}\right)^{2}+\frac{1}{r^{2}}\left(\eta \sigma_{3}+\xi \sigma_{2}\right)\right]\left(\begin{array}{c}
f_{m} \\
g_{m}
\end{array}\right)=0
$$

where

$$
\eta=s(m+\alpha)+\frac{1}{2}
$$

It is convenient to bring the matrix $\eta \sigma_{3}+\xi \sigma_{2}$ to diagonal form using

$$
\eta \sigma_{3}+\xi \sigma_{2}=\epsilon(\eta) \sqrt{\eta^{2}+\xi^{2}} U^{+} \sigma_{3} U
$$

where $\epsilon(x)$ is the usual alternating function $\epsilon(x)=x /|x|$ and the unitary matrix $U$ is

$$
U=\cos \frac{\theta}{2}-i \sigma_{1} \epsilon(\eta) \sin \frac{\theta}{2}
$$

with

$$
-\pi<\theta=\tan ^{-1} \frac{\xi}{\eta}<\pi
$$

By defining

$$
\chi_{m}=\left(\begin{array}{c}
\chi_{1 m} \\
\chi_{2 m}
\end{array}\right)=U\left(\begin{array}{c}
f_{m} \\
g_{m}
\end{array}\right)
$$

the second order equation for $\chi_{m}$ becomes

$$
\begin{aligned}
{\left[\frac{d^{2}}{d r^{2}}\right.} & +\frac{1}{r} \frac{d}{d r}-\frac{(m+\alpha)^{2}+\eta}{r^{2}}+E^{2} \\
& \left.-\left(M+\frac{\xi}{R}\right)^{2}+\frac{\epsilon(\eta) \sqrt{\eta^{2}+\xi^{2}}}{r^{2}} \sigma_{3}\right] \chi_{m}=0
\end{aligned}
$$

The solutions of Eq. (2.19) are

$$
\begin{aligned}
\chi_{1 m}=A_{m} e^{i k r}(-2 i k r)^{a_{m}} F\left(a_{m}+\frac{1}{2}+\frac{i M \xi}{k}\left|2 a_{m}+1\right|-2 i k r\right) \\
\quad+B_{m} e^{i k r}(-2 i k r)^{-a_{m}} F\left(-a_{m}+\frac{1}{2}+\frac{i M \xi}{k}\left|1-2 a_{m}\right|-2 i k r\right) \\
\chi_{2 m}=C_{m} e^{i k r}(-2 i k r)^{b_{m}} F\left(b_{m}+\frac{1}{2}+\frac{i M \xi}{k}\left|2 b_{m}+1\right|-2 i k r\right) \\
\quad+D_{m} e^{i k r}(-2 i k r)^{-b_{m}} F\left(-b_{m}+\frac{1}{2}+\frac{i M \xi}{k}\left|1-2 b_{m}\right|-2 i k r\right)
\end{aligned}
$$


where $F(a|c| z)$ is the usual confluent hypergeometric function and

$$
\begin{aligned}
& k^{2}=E^{2}-M^{2} \\
& a_{m}=\sqrt{\eta^{2}+\xi^{2}}-\frac{\epsilon(\eta)}{2} \\
& b_{m}=\sqrt{\eta^{2}+\xi^{2}}+\frac{\epsilon(\eta)}{2} .
\end{aligned}
$$

If one inverts Eq. (2.18), $f_{m}$ and $g_{m}$ are inferred to be

$$
\begin{aligned}
& f_{m}^{\text {out }}(r)=\cos \frac{\theta}{2} \chi_{1 m}+i \epsilon(\eta) \sin \frac{\theta}{2} \chi_{2 m} \\
& g_{m}^{\text {out }}(r)=i \epsilon(\eta) \sin \frac{\theta}{2} \chi_{1 m}+\cos \frac{\theta}{2} \chi_{2 m} .
\end{aligned}
$$

Before proceeding it is worthwhile to note the Galilean limit of Eq. (2.22). Since in that regime

$$
\begin{aligned}
& \cos \theta \rightarrow 1 \\
& \sin \theta \rightarrow 0 \\
& a_{m} \rightarrow|\eta|-\frac{\epsilon(\eta)}{2} \\
& b_{m} \rightarrow|\eta|+\frac{\epsilon(\eta)}{2},
\end{aligned}
$$

the Galilean limit of $f_{m}^{\text {out }}(r)$ is

$$
\begin{aligned}
f_{m}^{\text {out }}(r) & \rightarrow A_{m} e^{i k r}(-2 i k r)^{|\eta|-\frac{\epsilon(\eta)}{2}} \\
& F\left(|\eta|-\frac{\epsilon(\eta)}{2}+\frac{1}{2}+\frac{i M \xi}{k}\left|2\left(|\eta|-\frac{\epsilon(\eta)}{2}\right)+1\right|-2 i k r\right) \\
& +B_{m} e^{i k r}(-2 i k r)^{-|\eta|+\frac{\epsilon(\eta)}{2}} \\
& F\left(-|\eta|+\frac{\epsilon(\eta)}{2}+\frac{1}{2}+\frac{i M \xi}{k}\left|1-2\left(|\eta|-\frac{\epsilon(\eta)}{2}\right)\right|-2 i k r\right)
\end{aligned}
$$

By considering the four cases
(1) $s=1 \quad-\frac{1}{2}<m+\alpha<1$
(2) $s=-1 \quad-1<m+\alpha<\frac{1}{2}$
(3) $s=1 \quad-1<m+\alpha<-\frac{1}{2}$
(4) $s=-1 \quad \frac{1}{2}<m+\alpha<1$ 
respectively, one can show that for $|m+\alpha|<1$ Eq. (2.24) becomes

$$
\begin{aligned}
& A_{m} e^{i k r}(-2 i k r)^{|m+\alpha|} F\left(|m+\alpha|+\frac{1}{2}+\frac{i M \xi}{k}|1+2| m+\alpha||-2 i k r\right) \\
& \quad+B_{m} e^{i k r}(-2 i k r)^{-|m+\alpha|} F\left(-|m+\alpha|+\frac{1}{2}+\frac{i M \xi}{k}|1-2| m+\alpha||-2 i k r\right)
\end{aligned}
$$

as already derived in Ref. [7].

Before one considers the boundary condition at $r=R$, it is important to derive the relations between the coefficients $A_{m}, B_{m}, C_{m}$ and $D_{m}$ by using the first-order Dirac equation (2.1). From (2.1) the first-order equations for $f_{m}(r)$ and $g_{m}(r)$ are

$$
\begin{aligned}
\left(M-\frac{\xi}{r}-E\right) f_{m} & =i\left(\frac{d}{d r}+\frac{s(m+\alpha+s)}{r}\right) g_{m} \\
-\left(M-\frac{\xi}{r}+E\right) g_{m} & =i\left(\frac{d}{d r}-\frac{s(m+\alpha)}{r}\right) f_{m}
\end{aligned}
$$

which yield the coupled first order equations for $\chi_{1 m}$ and $\chi_{2 m}$

$$
\begin{aligned}
\frac{d \chi_{1 m}}{d r}+\left[\frac{\epsilon(\eta) \xi}{\sqrt{\eta^{2}+\xi^{2}}} M-\frac{\epsilon(\eta) \sqrt{\eta^{2}+\xi^{2}}-\frac{1}{2}}{r}\right] \chi_{1 m} \\
=i\left(E+M \frac{|\eta|}{\sqrt{\eta^{2}+\xi^{2}}}\right) \chi_{2 m} \\
\frac{d \chi_{2 m}}{d r}-\left[\frac{\epsilon(\eta) \xi}{\sqrt{\eta^{2}+\xi^{2}}} M-\frac{\epsilon(\eta) \sqrt{\eta^{2}+\xi^{2}}+\frac{1}{2}}{r}\right] \chi_{2 m} \\
=i\left(E-M \frac{|\eta|}{\sqrt{\eta^{2}+\xi^{2}}}\right) \chi_{1 m} .
\end{aligned}
$$

By inserting $\chi_{1 m}$ and $\chi_{2 m}$ into Eq. (2.26) the relations between coefficients are seen to be

$$
\begin{aligned}
& C_{m}=\Omega_{1} A_{m} \\
& D_{m}=\Omega_{2} B_{m}
\end{aligned}
$$

when $\epsilon(\eta)=1$, and

$$
\begin{aligned}
& A_{m}=\Omega_{1} C_{m} \\
& B_{m}=\Omega_{2} D_{m}
\end{aligned}
$$


when $\epsilon(\eta)=-1$ where

$$
\begin{aligned}
& \Omega_{1}=\frac{-k\left(\eta^{2}+\xi^{2}+M^{2} \xi^{2} k^{-2}\right)}{2\left(\eta^{2}+\xi^{2}\right)\left(2 \sqrt{\eta^{2}+\xi^{2}}+1\right)}\left(E+\frac{\eta}{\sqrt{\eta^{2}+\xi^{2}}} M\right)^{-1} \\
& \Omega_{2}=2 k\left(2 \sqrt{\eta^{2}+\xi^{2}}-1\right)\left(E+\frac{\eta}{\sqrt{\eta^{2}+\xi^{2}}} M\right)^{-1} .
\end{aligned}
$$

It can be shown by direct calculation that the $\xi \rightarrow 0$ limit of Eq. (2.27) is identical to the result obtained if one starts ab initio from the relativistic AB problem.

By using Eq. (2.27) the outside solution of $f_{m}(r)$ can be expressed as

$$
f_{m}^{\text {out }}(r)=\left\{\begin{array}{lll}
A_{m} u_{1}(r)+B_{m} u_{2}(r) & \text { when } & \epsilon(\eta)=1 \\
C_{m} v_{1}(r)+D_{m} v_{2}(r) & \text { when } & \epsilon(\eta)=-1
\end{array}\right.
$$

where

$$
\begin{aligned}
& u_{1}(r)=e^{i k r}\left[\cos \frac{\theta}{2}(-2 i k r)^{\beta_{m, s}} F\left(\beta_{m, s}+\frac{1}{2}+\frac{i M \xi}{k}\left|2 \beta_{m, s}+1\right|-2 i k r\right)\right. \\
&\left.+i \sin \frac{\theta}{2} \Omega_{1}(-2 i k r)^{\beta_{m, s}+1} F\left(\beta_{m, s}+\frac{3}{2}+\frac{i M \xi}{k}\left|2 \beta_{m, s}+3\right|-2 i k r\right)\right] \\
& u_{2}(r)=e^{i k r}\left[\cos \frac{\theta}{2}(-2 i k r)^{-\beta_{m, s}} F\left(-\beta_{m, s}+\frac{1}{2}+\frac{i M \xi}{k}\left|1-2 \beta_{m, s}\right|-2 i k r\right)\right. \\
&\left.+i \sin \frac{\theta}{2} \Omega_{2}(-2 i k r)^{-\beta_{m, s}-1} F\left(-\beta_{m, s}-\frac{1}{2}+\frac{i M \xi}{k}\left|-1-2 \beta_{m, s}\right|-2 i k r\right)\right] \\
& v_{1}(r)=e^{i k r}[ \cos \frac{\theta}{2} \Omega_{1}(-2 i k r)^{\beta_{m, s}+1} F\left(\beta_{m, s}+\frac{3}{2}+\frac{i M \xi}{k}\left|2 \beta_{m, s}+3\right|-2 i k r\right) \\
&\left.+i \sin \frac{\theta}{2}(-2 i k r)^{\beta_{m, s}} F\left(\beta_{m, s}+\frac{1}{2}+\frac{i M \xi}{k}\left|2 \beta_{m, s}+1\right|-2 i k r\right)\right] \\
&\left.\quad-i \sin \frac{\theta}{2}(-2 i k r)^{-\beta_{m, s}} F\left(-\beta_{m, s}+\frac{1}{2}+\frac{i M \xi}{k}\left|1-2 \beta_{m, s}\right|-2 i k r\right)\right] \\
& v_{2}(r)=e^{i k r}\left[\cos \frac{\theta}{2} \Omega_{2}(-2 i k r)^{\beta_{m, s}-1} F\left(-\beta_{m, s}-\frac{1}{2}+\frac{i M \xi}{k}\left|-2 \beta_{m, s}-1\right|-2 i k r\right)\right. \\
&
\end{aligned}
$$

and

$$
\beta_{m, s}=\sqrt{\eta^{2}+\xi^{2}}-\frac{1}{2}
$$


The boundary conditions at $r=R$

$$
\begin{aligned}
& \lim _{\varepsilon \rightarrow 0^{+}} f_{m}(R+\varepsilon)=\lim _{\varepsilon \rightarrow 0^{+}} f_{m}(R-\varepsilon) \\
& \lim _{\varepsilon \rightarrow 0^{+}} \frac{d f_{m}}{d r}(R+\varepsilon)=\lim _{\varepsilon \rightarrow 0^{+}}\left(\frac{d}{d r}+\frac{\alpha s}{R}\right) f_{m}(R-\varepsilon)
\end{aligned}
$$

give the ratios of the coefficients as

$$
\begin{aligned}
& \frac{A_{m}}{B_{m}}=\frac{J_{|m|}\left(k_{0} R\right) \frac{d u_{2}(R)}{d R}-u_{2}(R)\left(\frac{d}{d R}+\frac{\alpha s}{R}\right) J_{|m|}\left(k_{0} R\right)}{u_{1}(R)\left(\frac{d}{d R}+\frac{\alpha s}{R}\right) J_{|m|}\left(k_{0} R\right)-J_{|m|}\left(k_{0} R\right) \frac{d u_{1}(R)}{d R}} \\
& \frac{C_{m}}{D_{m}}=\frac{J_{|m|}\left(k_{0} R\right) \frac{d v_{2}(R)}{d R}-v_{2}(R)\left(\frac{d}{d R}+\frac{\alpha s}{R}\right) J_{|m|}\left(k_{0} R\right)}{v_{1}(R)\left(\frac{d}{d R}+\frac{\alpha s}{R}\right) J_{|m|}\left(k_{0} R\right)-J_{|m|}\left(k_{0} R\right) \frac{d v_{2}(R)}{d R}}
\end{aligned}
$$

for $\epsilon(\eta)=1$ and $\epsilon(\eta)=-1$ respectively, where $\frac{d}{d R} J_{|m|}\left(k_{0} R\right)$ is understood to be $\left[\frac{d}{d r} J_{|m|}\left(k_{0} r\right)\right]_{r=R}$. At this point it is appropriate to take the $R \rightarrow 0$ limit. In order to compare with various other $\mathrm{AB}$ problems the first and second leading terms of $u_{1}(r)$, $u_{2}(r), v_{1}(r)$ and $v_{2}(r)$ are given in Tables I, II, III, and IV respectively. From these tables it is seen that the Galilean limit of the exponents of $R$ for the first leading terms of $u_{2}(R)$ and $v_{1}(R)$ in the relativistic $\mathrm{ABC}$ problem do not coincide with those of the Galilean $\mathrm{ABC}$ problem. In other words, the Galilean limit operation does not commute with the $R \rightarrow 0$ limit. This feature makes the relativistic ABC problem quite different from the Galilean one. Another factor which exacerbates this difference is the $R$-dependence of $k_{0}$. While in the relativistic $\mathrm{ABC}$ problem $k_{0}$ is proportional to $R^{-1}$ for $R \rightarrow 0$, in the Galilean limit $k_{0}$ is proportional to $R^{-\frac{1}{2}}$. The first and second leading terms of $J_{|m|}\left(k_{0} R\right)$ and $\left(\frac{d}{d R}+\frac{\alpha s}{R}\right) J_{|m|}\left(k_{0} R\right)$ in the various AB problems are given in Table V and VI.

If one applies the first and second leading terms of the relativistic $\mathrm{AB}$ and Galilean $\mathrm{ABC}$ problems to Eq. (2.33), the conditions for the occurrence of a singular solution are

$$
\begin{aligned}
& |m+\alpha|<1 \\
& |m|+|m+\alpha|+\alpha s=0
\end{aligned}
$$

in the relativistic $\mathrm{AB}$ problem and

$$
\begin{aligned}
& |m+\alpha|<\frac{1}{2} \\
& |m|+|m+\alpha|+\alpha s=0
\end{aligned}
$$


for the Galilean ABC case. However, the nature of the relativistic ABC problem is vastly different. Of particular significance is the fact that for nonzero values of $\theta$ the functions $u_{1}, u_{2}, v_{1}$, and $v_{2}$ can each have both regular and irregular parts. By applying Eq. (2.33) one concludes that

$$
B_{m}=D_{m}=0
$$

so long as there is no fine tuning of $\xi$. Thus the radial solution becomes

$$
\begin{aligned}
f_{m}(r)=A_{m} e^{i k r}[ & \cos \frac{\theta}{2}(-2 i k r)^{\beta_{m, s}} F\left(\beta_{m, s}+\frac{1}{2}+\frac{i M \xi}{k}\left|2 \beta_{m, s}+1\right|-2 i k r\right) \\
& \left.+i \sin \frac{\theta}{2} \Omega_{1}(-2 i k r)^{\beta_{m, s}+1} F\left(\beta_{m, s}+\frac{3}{2}+\frac{i M \xi}{k}\left|2 \beta_{m, s}+3\right|-2 i k r\right)\right]
\end{aligned}
$$

for $\epsilon(\eta)=1$, and

$$
\begin{array}{r}
f_{m}(r)=C_{m} e^{i k r}\left[\cos \frac{\theta}{2} \Omega_{1}(-2 i k r)^{\beta_{m, s}+1} F\left(\beta_{m, s}+\frac{3}{2}+\frac{i M \xi}{k}\left|2 \beta_{m, s}+3\right|-2 i k r\right)\right. \\
\left.+i \sin \frac{\theta}{2}(-2 i k r)^{\beta_{m, s}} F\left(\beta_{m, s}+\frac{1}{2}+\frac{i M \xi}{k}\left|2 \beta_{m, s}+1\right|-2 i k r\right)\right]
\end{array}
$$

for $\epsilon(\eta)=-1$. From (2.36) bound state energies are obtained for the $\xi<0$ case by applying simultaneously the terminating condition to the two confluent hypergeometric functions. The result is

$$
k \equiv \sqrt{E^{2}-M^{2}}=-\frac{i M \xi}{n-1+\left[\eta^{2}+\xi^{2}\right]^{1 / 2}} \quad n=1,2, \ldots
$$

where use has been made of the fact that $\Omega_{1}$ vanishes in the $n=1$ state. Detailed discussion of this expression for the binding energies is deferred to the Conclusion.

\section{Scalar Coupling of the Discontinuous Coulomb Potential}

The most distinctive feature of the discontinuous Coulomb potential relative to the continuous one is that the electric field acquires a delta-function contribution which changes the boundary condition at $r=R$. Thus Eq. (2.7) in this case becomes

$$
E_{1} \pm i s E_{2}=e^{ \pm i s \phi}\left[\frac{\xi}{r^{2}} \theta(r-R)-\frac{\xi}{R} \delta(r-R)\right]
$$


while the boundary conditions at $r=R$ are

$$
\begin{aligned}
& \lim _{\varepsilon \rightarrow 0^{+}} f_{m}(R+\varepsilon)=\lim _{\varepsilon \rightarrow 0^{+}} f_{m}(R-\varepsilon) \\
& \lim _{\varepsilon \rightarrow 0^{+}} g_{m}(R+\varepsilon)=\lim _{\varepsilon \rightarrow 0^{+}} g_{m}(R-\varepsilon) \\
& \lim _{\varepsilon \rightarrow 0^{+}} \frac{d f_{m}}{d r}(R+\varepsilon)=\lim _{\varepsilon \rightarrow 0^{+}}\left(\frac{d}{d r}+\frac{\alpha s}{R}\right) f_{m}(R-\varepsilon)-\frac{i \xi}{R} g_{m}(R) .
\end{aligned}
$$

Since $g_{m}$ is included in the boundary conditions, one must solve for both $f_{m}(r)$ and $g_{m}(r)$ in the inside region. One readily establishes from the radial equation for the inside region that

$$
\begin{aligned}
& f_{m}^{\text {in }}(r)=E_{m} J_{|m|}(k r) \\
& g_{m}^{\text {in }}(r)=F_{m} J_{|m+s|}(k r)
\end{aligned}
$$

where

$$
k^{2}=E^{2}-M^{2}
$$

By using a first-order equation it is found that the relation between $E_{m}$ and $F_{m}$ can be written as

$$
F_{m}=i \epsilon(m s) \sqrt{\frac{E-M}{E+M}} E_{m}
$$

provided that $\epsilon(0)$ is taken to be +1 .

It is easily demonstrated that the outside solution is given by Eq. (2.29), just as in the case of the continuous Coulomb potential. By using the inside solutions (3.3) and the outside solutions (2.29) the boundary conditions (3.2) give the ratio of coefficients

$$
\frac{A_{m}}{B_{m}}=\frac{J_{|m|}(k R) \frac{d u_{2}(R)}{d R}-u_{2}(R) u(R)}{u_{1}(R) u(R)-J_{|m|}(k R) \frac{d u_{1}(R)}{d R}}
$$

for $\epsilon(\eta)=1$, and

$$
\frac{C_{m}}{D_{m}}=\frac{J_{|m|}(k R) \frac{d v_{2}(R)}{d R}-v_{2}(R) u(R)}{v_{1}(R) u(R)-J_{|m|}(k R) \frac{d v_{1}(R)}{d R}}
$$

for $\epsilon(\eta)=-1$ where

$$
u(R)=\left(\frac{d}{d R}+\frac{\alpha s}{R}\right) J_{|m|}(k R)+\frac{\xi}{R} \sqrt{\frac{E-M}{E+M}} \epsilon(m s) J_{|m+s|}(k R)
$$

In order to compare with the various $\mathrm{AB}$ problems the first and second leading terms of $u(R)$ in the $R \rightarrow 0$ limit are given in Table VII. It is important to note that if one 
substitutes these asymptotic forms for $u(R)$ into Eqs. (3.6) and (3.7) for the relativistic $\mathrm{AB}$ and Galilean ABC systems, Eq. (2.34) and (2.35) are again derived for the occurrence of singular solutions. In the relativistic ABC problem one again obtains Eqs. (2.37) and (2.38) if fine tuning of $\xi$ is not considered.

\section{Vector Coupling Theory}

In this section the results which have been derived in the preceding sections are extended to the vector coupling of a Coulomb potential in the relativistic AB problem. The Dirac equation to be discussed is

$$
\beta[M+\vec{\gamma} \cdot \vec{\Pi}] \psi=[E-V(r)] \psi
$$

which implies the second-order equation

$$
\begin{aligned}
{\left[\frac{\partial^{2}}{\partial r^{2}}\right.} & +\frac{1}{r} \frac{\partial}{\partial r}+\frac{1}{r^{2}}\left(\frac{\partial}{\partial \phi}+i \alpha\right)^{2} \\
& \left.+e s H \sigma_{3}+(E-V)^{2}-M^{2}-i\left(E_{1} \sigma_{1}+s E_{2} \sigma_{2}\right)\right] \psi=0
\end{aligned}
$$

By using Eq. (2.9) the radial equation for the continuous Coulomb potential is seen to be

$$
\begin{aligned}
\left\{\frac{d^{2}}{d r^{2}}\right. & +\frac{1}{r} \frac{d}{d r}-\frac{(m+\alpha)^{2}}{r^{2}}+\left[E-\frac{\xi}{r} \theta(r-R)-\frac{\xi}{R} \theta(R-r)\right]^{2}-M^{2} \\
& \left.-\frac{\frac{1}{2}-s(m+\alpha)}{r^{2}}\left(1-\sigma_{3}\right)-\frac{i \xi}{r^{2}} \sigma_{1} \theta(r-R)+e s H \sigma_{3}\right\}\left(\begin{array}{l}
f_{m} \\
g_{m}
\end{array}\right)=0 .
\end{aligned}
$$

From (4.3) the regular solution of $f_{m}$ in the inside region is

$$
f_{m}^{\text {in }}(r)=J_{|m|}\left(k_{0} r\right)
$$

where

$$
k_{0}=\left(E-\frac{\xi}{R}\right)^{2}-M^{2}
$$

In the outside region Eq. (4.3) can be written as

$$
\begin{aligned}
{\left[\frac{d^{2}}{d r^{2}}+\frac{1}{r} \frac{d}{d r}\right.} & -\frac{\eta+(m+\alpha)^{2}}{r^{2}}+\left(E-\frac{\xi}{r}\right)^{2} \\
& \left.-M^{2}+\frac{1}{r^{2}}\left(\eta \sigma_{3}-i \xi \sigma_{1}\right)\right]\left(\begin{array}{c}
f_{m} \\
g_{m}
\end{array}\right)=0
\end{aligned}
$$


Again one diagonalizes the $1 / r^{2}$ term using the result

$$
\eta \sigma_{3}-i \xi \sigma_{1}=\epsilon(\eta) \sqrt{\eta^{2}-\xi^{2}} U^{-1} \sigma_{3} U
$$

where

$$
\begin{aligned}
U^{ \pm 1} & =a_{+} \mp \epsilon(\eta) a_{-} \sigma_{2} \\
a_{ \pm} & = \pm \frac{1}{\sqrt{2}}\left[\frac{|\eta|}{\sqrt{\eta^{2}-\xi^{2}}} \pm 1\right]^{\frac{1}{2}}
\end{aligned}
$$

By defining

$$
\chi_{m} \equiv\left(\begin{array}{c}
\chi_{3 m} \\
\chi_{4 m}
\end{array}\right)=U\left(\begin{array}{l}
f_{m} \\
g_{m}
\end{array}\right)
$$

the second-order equations for $\chi_{3 m}$ and $\chi_{4 m}$ can be solved to yield

$$
\begin{aligned}
\chi_{3 m}= & A_{m} e^{i k r}(-2 i k r)^{c_{m}} F\left(c_{m}+\frac{1}{2}+\frac{i E \xi}{k}\left|2 c_{m}+1\right|-2 i k r\right) \\
& +B_{m} e^{i k r}(-2 i k r)^{-c_{m}} F\left(-c_{m}+\frac{1}{2}+\frac{i E \xi}{k}\left|-2 c_{m}\right|-2 i k r\right) \\
\chi_{4 m}= & C_{m} e^{i k r}(-2 i k r)^{d_{m}} F\left(d_{m}+\frac{1}{2}+\frac{i E \xi}{k}\left|2 d_{m}+1\right|-2 i k r\right) \\
& +D_{m} e^{i k r}(-2 i k r)^{-d_{m}} F\left(-d_{m}+\frac{1}{2}+\frac{i E \xi}{k}\left|1-2 d_{m}\right|-2 i k r\right)
\end{aligned}
$$

where

$$
\begin{aligned}
& c_{m}=\sqrt{\eta^{2}-\xi^{2}}-\frac{\epsilon(\eta)}{2} \\
& d_{m}=\sqrt{\eta^{2}-\xi^{2}}+\frac{\epsilon(\eta)}{2} .
\end{aligned}
$$

Upon obtaining the relations between coefficients

$$
\begin{aligned}
& C_{m}=\Omega_{3} A_{m} \\
& D_{m}=\Omega_{4} B_{m}
\end{aligned}
$$

for $\epsilon(\eta)=1$, and

$$
\begin{aligned}
& A_{m}=\Omega_{3} C_{m} \\
& B_{m}=\Omega_{4} D_{m}
\end{aligned}
$$


for $\epsilon(\eta)=-1$ where

$$
\begin{aligned}
& \Omega_{3}=\frac{-k\left(\eta^{2}-\xi^{2}+E^{2} \xi^{2} k^{-2}\right)}{2\left(\eta^{2}-\xi^{2}\right)\left(2 \sqrt{\eta^{2}-\xi^{2}}+1\right)}\left(E+\frac{\eta}{\sqrt{\eta^{2}-\xi^{2}}} M\right)^{-1} \\
& \Omega_{4}=2 k\left(2 \sqrt{\eta^{2}-\xi^{2}}-1\right)\left(E+\frac{\eta}{\sqrt{\eta^{2}-\xi^{2}}} M\right)^{-1}
\end{aligned}
$$

one can proceed to match the boundary conditions at $r=R$. This yields once again the result

$$
B_{m}=D_{m}=0
$$

in the absence of a fine tuning condition on $\xi$. Thus the radial wave equation becomes

$$
\begin{aligned}
f_{m}(r)=A_{m} e^{i k r}[ & a_{+}(-2 i k r)^{\gamma_{m, s}} F\left(\gamma_{m, s}+\frac{1}{2}+\frac{i M \xi}{k}\left|2 \gamma_{m, s}+1\right|-2 i k r\right) \\
& \left.\quad-a_{-} \Omega_{3}(-2 i k r)^{\gamma_{m, s}+1} F\left(\gamma_{m, s}+\frac{3}{2}+\frac{i M \xi}{k}\left|2 \gamma_{m, s}+3\right|-2 i k r\right)\right]
\end{aligned}
$$

for $\epsilon(\eta)=1$, and

$$
\begin{array}{r}
f_{m}(r)=C_{m} e^{i k r}\left[a_{+} \Omega_{3}(-2 i k r)^{\gamma_{m, s}+1} F\left(\gamma_{m, s}+\frac{3}{2}+\frac{i M \xi}{k}\left|2 \gamma_{m, s}+3\right|-2 i k r\right)\right. \\
\left.+i a_{-}(-2 i k r)^{\gamma_{m, s}} F\left(\gamma_{m, s}+\frac{1}{2}+\frac{i M \xi}{k}\left|2 \gamma_{m, s}+1\right|-2 i k r\right)\right]
\end{array}
$$

for $\epsilon(\eta)=-1$ where

$$
\gamma_{m, s}=\sqrt{\eta^{2}-\xi^{2}}-\frac{1}{2}
$$

In the case of the discontinuous Coulomb potential the radial wave function is once again given by Eqs. (4.14) and (4.15) in close analogy to the scalar coupling theory. In each case the bound state energies are found to be

$$
E_{V}^{2}=M^{2}\left[1+\frac{\xi^{2}}{\left[n-1+\sqrt{\left[s(m+\alpha)+\frac{1}{2}\right]^{2}-\xi^{2}}\right]^{2}}\right]^{-1} \quad n=1,2,3, \ldots
$$

This spectrum is in agreement with the result obtained in ref. 10 without reference to the vanishing radius flux tube method. 
It is of interest to note that the result (2.39) for the scalar binding energies $E_{S}$ and (4.17) can be combined in the single expression

$$
E_{\left(\begin{array}{l}
S \\
V
\end{array}\right)}=M\left\{1 \mp \frac{\xi^{2}}{\left[n-1+\left\{\left[s(m+\alpha)+\frac{1}{2}\right]^{2} \pm \xi^{2}\right\}^{\frac{1}{2}}\right]^{2}}\right\}^{ \pm \frac{1}{2}}
$$

This compact form allows one to discuss simultaneously the significance of the results obtained in the various coupling models considered here.

\section{Conclusion}

The method of the finite radius flux tube was advanced originally as a possible tool to deal with complications encountered in the relativistic AB calculation for spin-1/2 particles. It was successful in that application as well as in the corresponding Galilean ABC problem. In particular it was found [7] in the latter case that when the Coulomb potential is attractive bound state energies occur at

$$
\mathcal{E}_{n}=-\frac{1}{2} \frac{M \xi^{2}}{\left(n-\frac{1}{2} \pm|m+\alpha|\right)^{2}} \quad n=1,2, \ldots
$$

where the minus sign is realized only in the case $|m+\alpha|<\frac{1}{2}, \alpha s<0$. The corresponding result for the $\mathrm{ABCD}$ problem (i.e., the Dirac equation treatment of the $\mathrm{ABC}$ potential) is given by (4.18) and there arises the question as to the mutual consistency of these expressions.

Comparison of the two formulae in the Galilean limit indicates that compatibility requires that the form $\left|m+\alpha+\frac{s}{2}\right|-1$ of the $\mathrm{ABCD}$ case be equivalent to $\pm|m+\alpha|-$ $\frac{1}{2}$ of the $\mathrm{ABC}$ result. These are not obviously the same and in fact they cannot be generally equivalent. The expression (4.18) has the property that it is invariant under the replacement

$$
|m+\alpha| \rightarrow-|m+\alpha|-s \epsilon(m+\alpha)
$$

whereas (5.1) does not. More simply, for $\alpha=0$ the nonrelativistic energies (5.1) become spin independent unlike the relativistic ones. More detailed analysis of the ABCD spectrum shows that even though there are singular states in the general case (corresponding to the 
minus sign choice in (5.1)), the details of the spectrum do not agree in the limit in which $c$ (the velocity of light) becomes arbitrarily large.

It is not difficult to trace the source of this discrepancy. As has been emphasized during the course of the calculations presented here, the very different qualitative behaviors of the Galilean and the relativistic wave functions for $\xi \neq 0$ mean that the $R \rightarrow 0$ and $c \rightarrow \infty$ limits do not commute. Thus the singular solutions which arise in the Galilean limit from the delta function magnetic field are in the ABCD calculation a result of purely relativistic effects in the $\xi \neq 0$ case. In fact the magnetic field term had no effect whatever upon the structure of the relativistic wave functions in the $R=0$ limit.

There is, of course, no a priori requirement that a relativistic wave equation give totally satisfactory results in describing a physical phenomenon which presumably demands a field theoretical approach for full consistency. The strains put upon wave mechanics by such phenomena as Klein's paradox are, for example, well known. On the other hand one generally expects relativistic wave equations to give reliable results in the Galilean limit. That has not happened in the present case for reasons which have been carefully documented. Whether the shortcoming is in the finite radius flux tube method or in the nature of relativistic wave equations - or even whether it makes sense to attempt to separate these two issues - is not obvious. This paper does, however, clearly show that one is now pressing hard upon the limits of joint applicability of these two calculational techniques.

\section{Acknowledgment}

This work is supported in part by the U.S. Department of Energy Grant No. DE-FG02-91ER40685. D.K.P. would like to thank the Korean Science and Engineering Foundation. 


\section{References}

1. Y. Aharonov and D. Bohm, Phys. Rev. 115, 485 (1959).

2. M. Peshkin and A. Tonomura, The Aharonov-Bohm Effect, (Springer-Verlag, Berlin, 1989).

3. F. Wilczek, Phys. Rev. Lett. 48, 1144 (1982).

4. Ph. de Sousa Gerbert, Phys. Rev. D40, 1346 (1989).

5. C.R. Hagen, Phys. Rev. Lett. 64, 503 (1990), Int. J. Mod. Phys. A6, 3119 (1991).

6. F.A.B. Coutinho and J.F. Perez, Phys. Rev. D48, 932 (1993).

7. C.R. Hagen, Phys. Rev. D48, 5935 (1993).

8. J.M. Lévy-Leblond, Commun. Math. Phys. 6, 286 (1967).

9. D.K. Park, hep-th9405009 (1994).

10. S.H. Guo, X.L. Yang, F.T. Chan, K.W. Wong, and W.Y. Ching, Phys. Rev. A43, 1197 (1991). 
TABLE I. Leading terms in $u_{1}(R)$.

\begin{tabular}{|l|l|c|}
\hline$u_{1}(R)(\epsilon(\eta)=1)$ & First leading term & Second leading term \\
\hline Relativistic ABC & $\cos \frac{\theta}{2}(-2 i k R)^{\beta_{m, s}}$ & $(-2 i k R)^{\beta_{m, s}+1}\left[\begin{array}{c}\frac{(i M \xi / k)}{1+2 \beta_{m, s}} \\
+i \sin \frac{\theta}{2} \Omega_{1}\end{array}\right]$ \\
\hline Relativistic AB $\frac{\theta}{2}$ \\
\hline Galilean ABC & $(-2 i k R)^{s(m+\alpha)}$ & $O\left(R^{s(m+\alpha)+2}\right)$ \\
\hline
\end{tabular}

TABLE II. Leading terms in $u_{2}(R)$.

\begin{tabular}{|l|l|c|}
\hline$u_{2}(R)(\epsilon(\eta)=1)$ & \multicolumn{1}{|c|}{ First leading term } & Second leading term \\
\hline Relativistic ABC & $i \sin \frac{\theta}{2} \Omega_{2}(-2 i k R)^{-\beta_{m, s}-1}$ & $(-2 i k R)^{-\beta_{m, s}}\left[\begin{array}{c}\cos \frac{\theta}{2} \\
+\frac{M \xi / k}{1+2 \beta_{m, s}} \sin \frac{\theta}{2} \Omega_{2}\end{array}\right]$ \\
\hline Relativistic AB & $(-2 i k R)^{-s(m+\alpha)}$ & $O\left(R^{-s(m+\alpha)+2}\right)$ \\
\hline Galilean ABC & $(-2 i k R)^{-s(m+\alpha)}$ & $\frac{i M \xi / k}{1-2 s(m+\alpha)}(-2 i k R)^{-s(m+\alpha)+1}$ \\
\hline
\end{tabular}


TABLE III. Leading terms in $v_{1}(R),\left[\Omega_{1}^{G}\right.$ is Galilean limit of $\left.\Omega_{1}\right]$.

\begin{tabular}{|l|l|c|}
\hline$v_{1}(R)(\epsilon(\eta)=-1)$ & \multicolumn{1}{|c|}{ First leading term } & Second leading term \\
\hline Relativistic ABC & $-i \sin \frac{\theta}{2}(-2 i k R)^{\beta_{m, s}}$ & $(-2 i k R)^{\beta_{m, s}+1}\left[\begin{array}{c}\cos \frac{\theta}{2} \Omega_{1} \\
+\frac{M \xi / k}{1+2 \beta_{m, s}} \sin \frac{\theta}{2}\end{array}\right]$ \\
\hline Relativistic AB & $\Omega_{1}(\xi=0)(-2 i k R)^{-s(m+\alpha)}$ & $O\left(R^{-s(m+\alpha)+2}\right)$ \\
\hline Galilean ABC & $\Omega_{1}^{G}(-2 i k R)^{-s(m+\alpha)}$ & $\Omega_{1}^{G}(-2 i k R)^{-s(m+\alpha)+1} \frac{i M \xi / k}{1-2 s(m+\alpha)}$ \\
\hline
\end{tabular}

TABLE IV. Leading terms in $v_{2}(R),\left[\Omega_{2}^{G}\right.$ is Galilean limit of $\left.\Omega_{2}\right]$.

\begin{tabular}{|l|l|c|}
\hline$v_{2}(R)(\epsilon(\eta)=-1)$ & \multicolumn{1}{|c|}{ First leading term } & Second leading term \\
\hline Relativistic ABC & $\cos \frac{\theta}{2} \Omega_{2}(-2 i k R)^{-\beta_{m, s}-1}$ & $-(-2 i k R)^{-\beta_{m, s}}\left[\begin{array}{c}\frac{i M \xi / k}{1+2 \beta_{m, s}} \cos \frac{\theta}{2} \Omega_{2} \\
+i \sin \frac{\theta}{2}\end{array}\right]$ \\
\hline Relativistic AB & $\Omega_{2}(\xi=0)(-2 i k R)^{s(m+\alpha)}$ & $O\left(R^{s(m+\alpha)+2}\right)$ \\
\hline Galilean $\mathrm{ABC}$ & $\Omega_{2}^{G}(-2 i k R)^{s(m+\alpha)}$ & $(-2 i k R)^{s(m+\alpha)+1} \frac{i M \xi / k}{1+2 s(m+\alpha)} \Omega_{2}^{G}$ \\
\hline
\end{tabular}


TABLE V. Leading terms in $J_{|m|}\left(k_{0} R\right)\left[k_{0}^{G}=\left[2 m\left(E-\frac{\xi}{R}\right)\right]^{1 / 2}\right]$.

\begin{tabular}{|l|l|c|}
\hline \multicolumn{1}{|c|}{$J_{|m|}\left(k_{0} R\right)$} & First leading term & Second leading term \\
\hline Relativistic ABC & $i^{|m|} I_{|m|}(\xi)$ & $i^{|m|} M I_{|m|}^{\prime}(\xi) R$ \\
\hline Relativistic AB & $\frac{\left(\frac{\sqrt{E^{2}-M^{2}}}{2}\right)^{|m|}}{\Gamma(1+|m|)} R^{|m|}$ & $-\frac{\left(\frac{\sqrt{E^{2}-M^{2}}}{2}\right)^{|m|+2}}{\Gamma(2+|m|)} R^{|m|+2}$ \\
\hline Galilean ABC & $\frac{\left(k_{0}^{G} R\right)^{|m|}}{2^{|m|} \Gamma(1+|m|)}$ & $-\frac{\left(k_{0}^{G} R\right)^{|m|+2}}{2^{|m|+2} \Gamma(2+|m|)}$ \\
\hline
\end{tabular}

TABLE VI. Leading terms in $\left(\frac{d}{d R}+\frac{\alpha s}{R}\right) J_{|m|}\left(k_{0} R\right)$.

\begin{tabular}{|l|c|c|}
\hline$\left(\frac{d}{d R}+\frac{\alpha s}{R}\right) J_{|m|}\left(k_{0} R\right)$ & First leading term & Second leading term \\
\hline Relativistic ABC & $i^{|m| \frac{(|m|+\alpha s) I_{|m|}(\xi)+\xi I_{|m|+1}(\xi)}{R}}$ & $i^{|m|} M\left(\begin{array}{c}(|m|+\alpha s) I_{|m|}^{\prime}(\xi) \\
+I_{|m|+1}(\xi) \\
+\xi I_{|m|+1}^{\prime}(\xi)\end{array}\right)$ \\
\hline Relativistic AB & $(|m|+\alpha s) \frac{\left(\frac{\sqrt{E^{2}-M^{2}}}{2}\right)^{|m|}}{\Gamma(1+|m|)} R^{|m|-1}$ & $O\left(R^{|m|+1}\right)$ \\
\hline Galilean ABC & $(|m|+\alpha s) \frac{\left(k_{0}^{G}\right)^{|m|}}{2^{|m|} \Gamma(1+|m|)} R^{|m|-1}$ & $O\left(\left(k_{0}^{G}\right)^{|m|+2} R^{|m|+1}\right)$ \\
\hline
\end{tabular}


TABLE VII. Leading terms in $u(R)$.

\begin{tabular}{|c|c|c|}
\hline$u(R)$ & First leading term & Second leading term \\
\hline $\begin{array}{c}\text { Relativistic ABC } \\
(m s \geq 0)\end{array}$ & $\frac{k^{|m|} R^{|m|-1}}{2^{|m|} \Gamma(1+|m|)}(|m|+\alpha s)$ & $\xi \sqrt{\frac{E-M}{E+M}} \frac{k^{|m|+1} R^{|m|}}{2^{|m|+1} \Gamma(2+|m|)}$ \\
\hline $\begin{array}{c}\text { Relativistic ABC } \\
(m s<0)\end{array}$ & $-\xi \sqrt{\frac{E-M}{E+M}} \frac{k^{|m|-1} R^{|m|-2}}{2^{|m|-1} \Gamma(|m|)}$ & $\frac{k^{|m|} R^{|m|-1}}{2^{|m|} \mid(1+|m|)}(|m|+\alpha s)$ \\
\hline Relativistic AB & $\frac{k^{|m|} R^{|m|-1}}{2^{|m|} \Gamma(1+|m|)}(|m|+\alpha s)$ & $O\left(R^{|m|+1}\right)$ \\
\hline Galilean ABC & $\frac{k^{|m|} R^{|m|-1}}{2^{|m|} \Gamma(1+|m|)}(|m|+\alpha s)$ & $O\left(R^{|m|+1}\right)$ \\
\hline
\end{tabular}

